\title{
Generador de preguntas dinámicas en tiempo real para las MYPE, capturando datos del cliente en los formularios de pedido, consulta y reclamos, explotando la información utilizando árboles de decisión
}

\author{
Generator of dynamic questions in realtime for the MYPE, \\ capturing the client's data in the order forms consults and \\ birdcalls, exploiting the information using trees of decision
}

\author{
Karem Jaquelin Peralta Venegas
}

http://dx.doi.org/10.21503/CienciayDesarrollo.2010.v11.11

\section{RESUMEN}

Hoy por hoy, las MYPE comercializadoras necesitan un cambio de estrategia, centrada en los clientes y ya no sólo en los productos, por dos razones: los productos de calidad estándar y el mercado amplio globalizado; por tanto, necesitan adoptar una estrategia centrada en el cliente, pero en el mercado no existen herramientas tecnológicas adecuadas a las MYPE, que sean de fácil uso, de bajo costo y que integren técnicas de bussines intelligence que les ayuden a adoptar esta nueva estrategia; ya que sólo cuentan con sistemas transaccionales de compras y ventas. Por tal razón se propone una herramienta con todas las características anteriores, construyendo un generador de preguntas dinámicas para la captura de datos del cliente y, la explotación de ésta, usando herramientas de bussiness intelligence como los cubos OLAP y el Algoritmo de árboles de decisión.

Palabras clave: MYPE, generador de preguntas, cubos OLAP, algoritmo de árbol de decisión, CRM.

\section{ABSTRACT}

Today MYPE trading need a change of customer-focused strategy and not just the products for two reasons: the products of standard quality and extensive global market therefore need to adopt a customer-focused strategy, but the market does not exist appropriate technological tools to MYPE, that are easy to use, affordable and integrated Business Intelligence techniques to help them adopt this new strategy; as transactional systems have only purchases and sales. For this reason it is proposed a tool with all the above characteristics, building a generator of dynamic queries to capture customer data and operate this business intelligence using tools such as OLAP cubes and the decision tree algorithm.

Key words: $M Y P E$, generator of questions, cubes OLAP, algorithm of tree of decision, CRM.

\footnotetext{
* Alumna de la Escuela Académico-Profesional de Ingeniería de Sistemas e Informática, Filial Arequipa.
} 


\section{INTRODUCCIÓN}

En la actualidad, las microempresas y pequeñas empresas constituyen las principales generadoras de empleo en el Perú. Por ello, el presente trabajo está orientado a que el empresario de las PYME se introduzca en el campo de la administración mediante la identificación de clientes. Es importante conocer al cliente, ya que actualmente se le considera como "la razón de ser" del negocio, y no como anteriormente se consideraba, el producto. Las herramientas tecnológicas existentes en el mercado para estudiar al cliente son muy complejas y muy costosas, quedando fuera del alcance de los microempresarios y pequeñas empresas. Con la aplicación de un generador de preguntas dinámicas de bajo costo y de fácil acceso, y el uso de técnicas de bussines intelligence, las MYPE podrán introducirse en el mundo del marketing, iniciando su estudio al cliente mediante la captura y explotación de datos en las fase de venta ypostventa.

\section{Problema de la investigación}

Las MYPE tienen las mismas necesidades de análisis de información del cliente que las empresas de gran tamaño; sin embargo, tienen muchas más desventajas y limitaciones que estas últimas.

El problema principal es que no existen herramientas tecnológicas adecuadas para las MYPE en el mercado, debido a que estas son complejas en su manejo y aun costosas. Además, debería existir una herramienta tecnológica adecuada a las MYPE que capture los datos del cliente e integre las técnicas de bussines intelligence para explotar la información. Este problema podría resumirse en la siguiente interrogación: ¿Cómo obtener la información del cliente usando una herramienta adecuada a las MYPE que integre los procesos transaccionales con la captura de la información del cliente, y a la vez explote la información capturada utilizando alguna técnica de business intelligence?

\section{Objetivo de la investigación}

Construir un generador de preguntas dinámicas de bajo costo y fácil uso, que capture la información del cliente durante los procesos transaccionales de pedidos, consultas, reclamos y permita explotar la información, utilizando árboles de decisión. A la vez, se propone agrupar la información empleando una herramienta de bussines intelligence que sea accesible para las MYPE comercializadoras dedicadas a la venta de productos.

\section{Justificación e importancia}

Actualmente, las MYPE tienen la necesidad de un cambio de estrategia por dos razones: por los productos estandarizados y por la multioferta. Por ello, ahora las MYPE, necesitan centrarse en el cliente con ayuda de herramientas tecnológicas. Gracias a la construcción de un generador de preguntas dinámicas de bajo costo y fácil uso, y el uso de cubos OLAP y el algoritmo de clasificación C4.5, las MYPE comercializadoras podrán obtener un cambio de estrategia, centrada en el cliente, y contribuir a su inserción en el mercado competitivo.

$\mathrm{Su}$ importancia radica en que no existen sistemas orientados al cliente adecuadas a las MYPE, ya que los sistemas existentes son complejos de manejary tienen costosinaccesibles para las MYPE. El sistema propuesto ayudará a las MYPE comercializadoras a capturar la información del cliente y así obtener un cambio de estrategia orientado al cliente, aplicando la técnica Target Marketing del CRM para su crecimiento. 


\section{MATERIAL Y MÉTODO}

\section{Descripción de la solución}
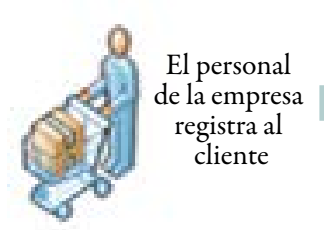

Cliente

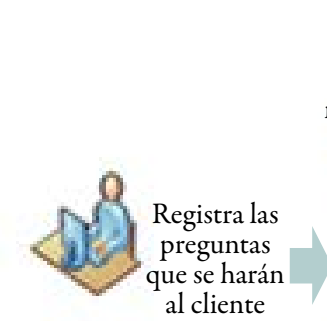

Administrador
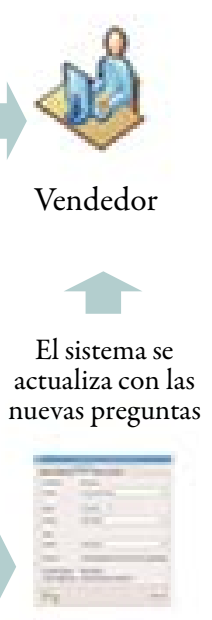

MS SQL
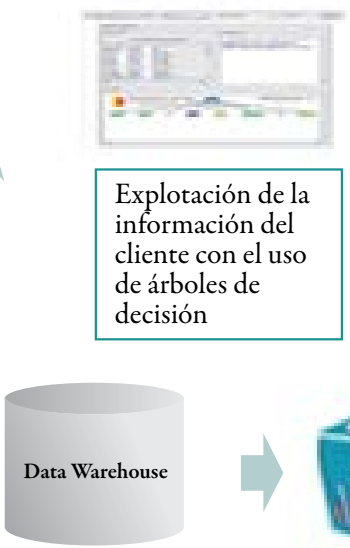

Almacena la información concerniente al cliente
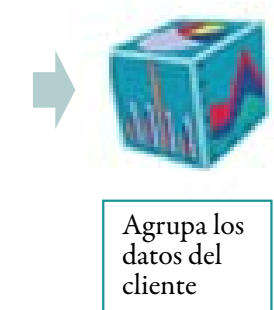

Administrador Dueño

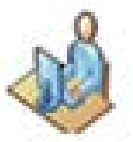

\section{Figura 1. Esquema conceptual del sistema}

El administrador de la MYPE se encarga de incorporar preguntas dinámicas al sistema transaccional de compras y ventas, en el cual puede modificar, eliminar e ingresar preguntas en tiempo real, de tal manera que, cuando llegue el cliente, el vendedor, además de atender las compras, registra las respuestas del cliente en el sistema. El administrador, luego de obtener la información del cliente, puede explotarla haciendo uso de cubos OLAP para la toma decisiones. Además, el sistema incorpora el algoritmo c4.5 de árboles de decisiones para agrupar la información capturada, permitiéndole conocer las respuestas de los clientes con sus respectivos correos, para enviarles publicidad dirigida de acuerdo con sus necesidades (ver Figura 1).

\section{Requisitos funcionales}

- Permitir ingresar datos del cliente.

- Permitir el mantenimiento de preguntas dinámicas.
- Asociar las preguntas a los controles determinados.

- Mostrar información utilizando cubos OLAP

- Explotar la información del cliente utilizando árboles de decisión.

El sistema propuesto no requiere de un personal muy capacitado, ya que, como vemos en el diagrama (ver Figura 2), el vendedor de la MYPE, a la vez que registra las ventas, consulta los reclamos del cliente, registra la información del cliente. Luego, el administrador-dueño de la MYPE puede tomar decisiones pertinentes con la ayuda de cubos OLAP o con el algoritmo del árbol de decisión propuesto.

\section{RESULTADOS OBTENIDOS}

Los resultados de la tasa de efectividad de orden del sistema generador de preguntas dinámicas visualizando y explotando la 


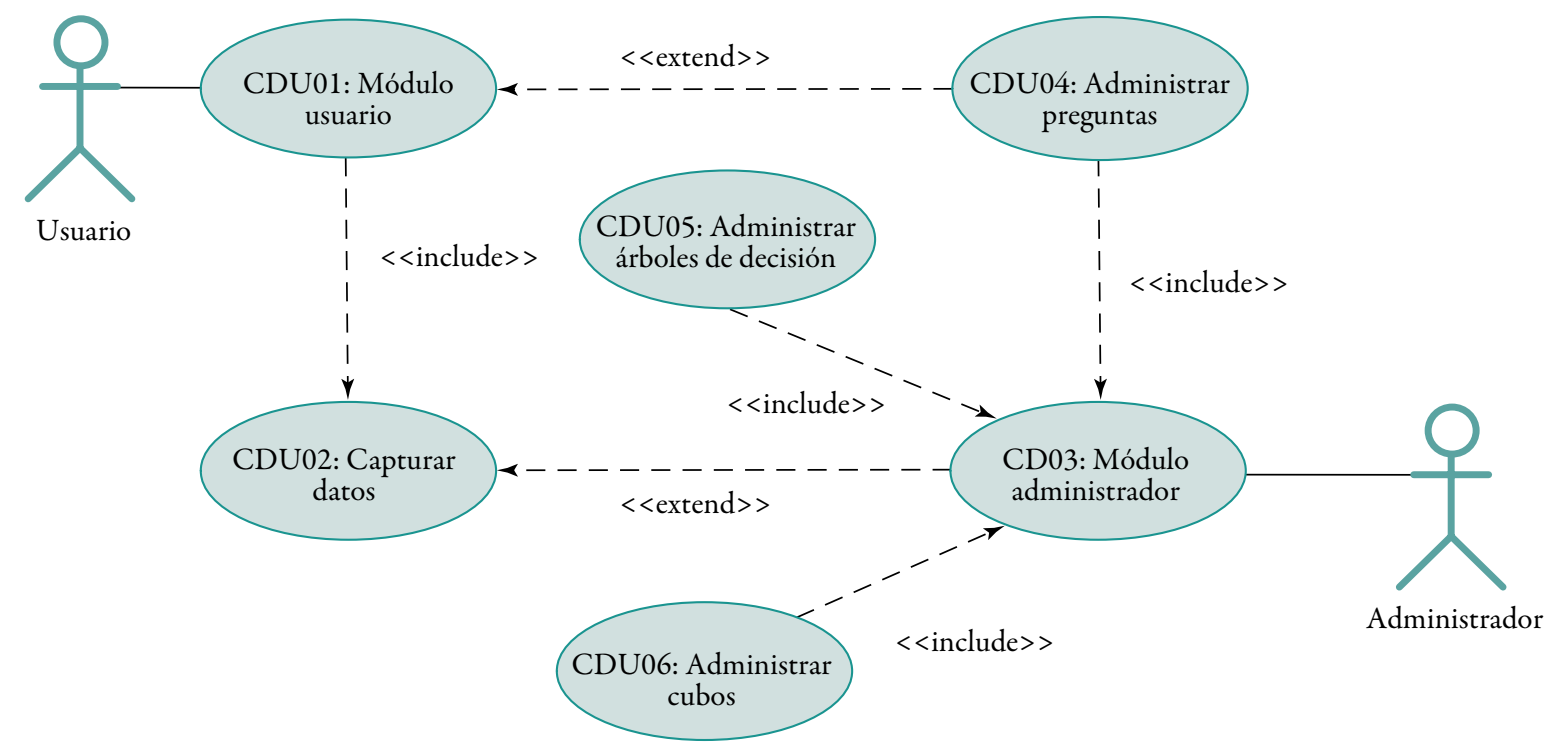

Figura 2. Sistema generador de preguntas dinámicas de la información del cliente, además de su visualización y explotación

información es de 0,7111167. Lo cual nos hace referencia a un $71,112 \%$ de efectividad del sistema.

Se logró construir un generador de preguntas dinámicas de bajo costo y fácil uso que captura la información del cliente durante los procesos transaccionales de pedidos, consultas, reclamos con lo que luego se explota la información, utilizando para ello los árboles de decisión propuestos y cubos OLAP.

\section{CONCLUSIONES}

- Actualmente la herramienta captura los datos del cliente en sus transacciones.

- Este prototipo tiene la facilidad de modificar, ingresar, eliminar las preguntas según el interés de la MYPE para almacenar información relevante a los objetivos de la MYPE.

- Este prototipo permite que la MYPE ya no solo se oriente al producto sino al cliente.

- Este prototipo está diseñado para un uso de fácil manejo y tiene un bajo costo para la MYPE.
- El uso de cubos OLAP facilita la agrupación de la información del cliente, tomada en cuenta desde diferentes perspectivas.

- Se comprobó que las MYPE pueden contar con herramientastecnológicas para el estudio del cliente, contribuyendo al crecimiento de la empresa en el actual mercado de la multioferta y de productos estandarizados.

- Se utilizó el algoritmo de árboles de decisión C4.5 para la explotación de la información recolectada del cliente, por cuanto resulta de un bajo costo y fácil uso.

- Gracias al algoritmo de árboles de decisión C4.5, se pudo orientar la investigación hacia la técnica Target Marketing del CRM para enviar publicidad dirigida a los clientes.

\section{TRABAJOS FUTUROS}

Investigación para construir una herramienta completa adecuada a la MYPE equivalente a un sistema CRM. 


\section{REFERENCIAS BIBLIOGRÁFICAS}

1. Wikilearning. Diferencias entre datawarehouse y base de datos operacionales (OLTP), en: bttp://www.wikilearning.com/apuntes data_warehouse-que_es_un_datawarebouse/23032-1, Giulio, 1 de mayo 2007.

2. Dybox Information Technologi. "Cubos Online Analytival Processing", en: http:// www.dybox.cl/pdf/cubos.PDF, Santiago, Chile, 2008.

3. Comercio de organizaciones privadas de promoción al desarrollo de la pequeña y microempresa, "Adaptación de las nuevas estrategias de autofinanciamiento a las nuevas condiciones de Mercado", en: http://www.nesst.org/Espanol/documents/ COPEMESPFINAL.pdf

4. ImhoffClaudia,Loftis Lisa, Geiger Jonathan. Building the Customer - Centric Enterprise, Data Warehousing Techniques for Supporting Customer Relationship Management. Editorial Wiley. Primera edición. Estados Unidos, 2001.

5. Curry Jay, Curry Adam, CRM - Customer Relationship Managemen. Cómo implementar y beneficiarse de la gestión de las relaciones con los clientes". Ediciones Gestión 2000, SA. Primera edición en español. España, 2002. 